\title{
MarkerClock: A Communicating Augmented Clock for Elderly
}

\author{
Yann Riche ${ }^{1,2}$ and Wendy Mackay ${ }^{1}$ \\ ${ }^{1}$ INRIA/LRI and Univ. Paris-Sud, France \\ ${ }^{2}$ Univ. of Queensland, Australia \\ yann.riche@lri.fr, wendy.mackay@inria.fr
}

\begin{abstract}
This paper presents markerClock, a communication appliance embedded into a clock and designed for seniors as a simple and intuitive device. MarkerClock enhances seniors' connectedness to their social networks, particularly friends, neighbors and relatives, therefore increasing the potential of human communication for providing and receiving care. In doing so, markerClock supports reciprocal care behaviors observed during our initial user study, which could be used to leverage the need for institutionalized care. This paper describes markerClock and its implementation of both passive and active communications.
\end{abstract}

\section{Introduction}

In industrialized countries, the population is aging [1, forcing institutions to seek innovative ways to address this challenge. To date, many researchers are exploring how monitoring solutions fitted into our seniors' homes can leverage the stress on care facilities. Monitoring technologies are designed to support the caregivers' tasks - help the helper - by sensing the seniors' environment. However, monitoring systems are invasive solutions, often ignoring simpler ways in which technology can benefit a larger population, such as maintaining social connectedness and preventing isolation and loneliness. In this context, our project explores how communication appliances (CA) [2] can help seniors to better age in place while offering an alternative to monitoring care solutions.

\section{Related Work}

Our preliminary study 3] highlighted the role of peer support amongst seniors. Subsequently, we define PeerCare as the bilateral, reciprocal care relation amongst seniors. In this context, our approach extends current and past research on media spaces 4 and home computer mediated communication (CMC) to design a communication appliance 2] supporting the well-being of seniors aging in place. Communication appliances (CA) are defined as "[...]simple-to-use, single-function devices that let people communicate, passively or actively, via some medium, with one or more remotely-located friends or family" 2]. In particular, CA can establish shared awareness between relatives and friends through 
the use of both passive and active communication. While home is becoming one of the major markets for interactive systems, it presents specific challenges by shifting the designer's focus from productivity to more intimate concerns such as closeness, intimacy, leisure, or entertainment. Recently, the interLiving project 5] studied the design of CA for intergenerational home communication and provided a context and a set of methods to study and design $\mathrm{CMC}$ in the home.

\section{MarkerClock}

MarkerClock (Fig. 2) is specifically designed to address the needs of the seniors by augmenting a simple clock into a communicating device. Communications are drawn around the clock as concentric colored spirals, each representing a different user. As a CA, markerClock supports active communication by using symbols, and passive communication by sharing activity values. Passive communication is a trace of motion captured by a camera fitted on the clock. Five levels of motion are visible (Fig. 1.b) from no motion (very light color) to extreme motion (very dense color). The motion trace does not require explicit user actions and provides peripheral communication among users. At the same time, the data exchanged is very limited and provides very little information, thus protecting the privacy of each participant. Moreover, the motion detection feature only captures motion occurring in the field of view of the camera. This also reinforces privacy protection since the data collection occurs only in a specific area decided by the user. Users can cheat and move the camera away, and decide whether to be in the field of view of the camera or not.

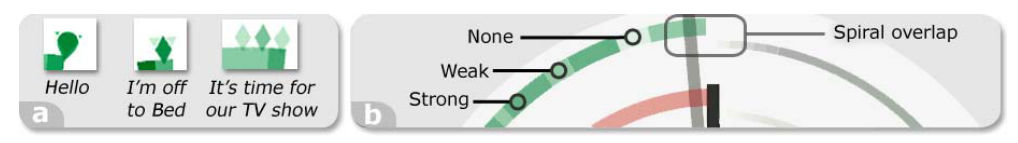

Fig. 1. Examples of a) users' codes, b) user's motion trace

Simple symbols, shaped to point at a particular spot on the motion trace, are used for active communication. By using the inherent time mapping of the clock, the time of the messages becomes explicit. For example, if Beatrice sends a droplet every morning to say she is all right, the same symbol sent at $8 \mathrm{pm}$ can convey a different meaning. Symbols are also explicitly chosen to carry no predefined meaning. Users are expected to build their own meaning and extend the available vocabulary using combinations of symbols (Fig. 1.a). The message is only explicit amongst people having the knowledge of the code and thus becomes part of the appropriation of the clock.

\subsection{An Augmented Clock}

While many existing products add extra functionalities to their designs, markerClock is a selection of features chosen for their simplicity and usefulness. Clocks 
are particularly suited to be augmented as they are ubiquitous in our homes. MarkerClock remains a simple clock and reuses existing interactions. Users can browse past communication by simply moving the hour hand clockwise or anticlockwise. The time and date of the current focus is then displayed as text at the bottom left corner of the clock. Users can return to the present by either bringing the hands back to the present time or by waiting 3 seconds without touching the clock.

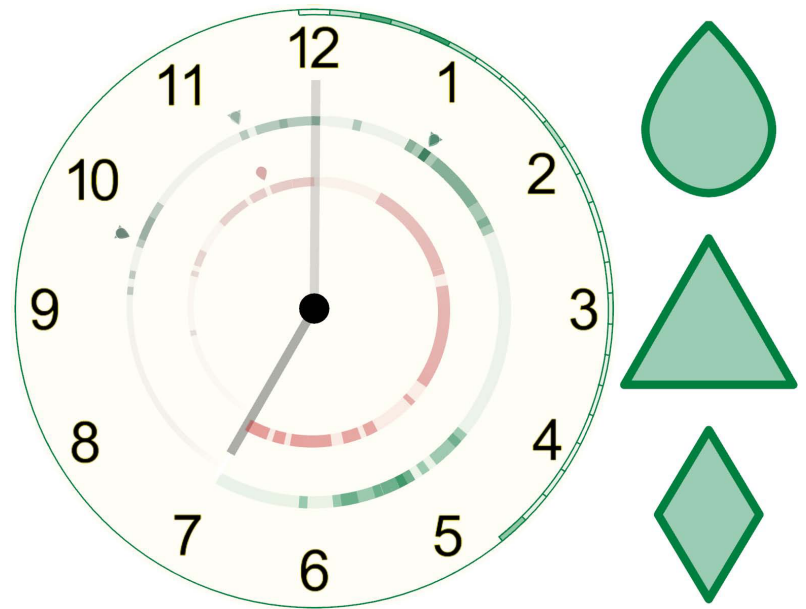

Fig. 2. Preview of a markerClock showing two connected users

The symbols available for active communication are displayed on the side of the clock. They are large enough to accommodate impaired eyesight and motor skills. Each user is also identified by his color, which is used for displaying his communication across connected clocks. The clock displays each message sent at the position of the hour hand for that time. For example messages sent at $3 \mathrm{pm}$ will be displayed in front of the number 3 on the clock. The thickness of the spiral depiciting motion decreases over time so as to easily distinguish between present and past communication. The age of a symbol or portion of motion trace is also emphasized by decreasing their saturation as time advances. The half hour of activity also fades to disappear totally (See Fig. 1.b). MarkerClock provides similar representations of self and other users on each device. A clock can display between 1 and 4 users, which can be separated into groups so that the friends do not see what the neighbor sends. This is particularly important as the social networks of seniors are often made of many sub-networks, which do not necessarily communicate with each other.

\subsection{Routines and Temporal Context}

The history of the past 12 hours of activity and the exploration of past communications allow users to build a shared knowledge of each other's routines. Users 
can compare the existing traces and their intimate knowledge to interpret events and stay aware of each other's activities. For example, Marie's brother, Sam, is used to see her activity start before 9am every morning. If this does not happen one morning, he might call her to check that everything is right. Knowledge of particular events is already shared by usual means such as phone or emails, if that change was to be expected, we assume that the information was passed during other regular communications.

By using the familiar mapping between time and geometry, markerClock enhances the role of time in messages and makes the "historical" context important. This use of time to provide explicit context for communication extends existing asynchronous/synchronous paradigms found in the literature. For example, If the clock is situated in her friend's living room, Beatrice will know that if a motion occurred between 4 and 5am, this is not only a night snack. This may show a sleep disorder due to her friend's upcoming surgery. Beatrice can clearly see when her friend was up during the night because the information is not lost (the old information is replaced by a newer one) and is readily available.

\section{Conclusion}

MarkerClock is an augmented clock built upon seniors' stories of their daily life. It uses simple active communication and non intrusive passive communication to support shared awareness amongst seniors. The evaluation of markerClock has been initiated by its deployment in our lab between people located in different offices and its introduction to a few seniors. These initial stages of evaluation allowed the refinement of some details such as color contrasts, decrease of motion sampling frequency, and the suggestion of tactile interaction. A deployment in situ will allow the contextual evaluation of markerClock. Results will be used to design new communication appliances and to refine markerClock itself.

\section{References}

[1] UN: World population and world urbanization prospects (2005)

[2] Mackay, W., Riche, Y., Labrune, J.-B.: Communication appliances: Shared awareness for intimate social networks. In: ACM SIGCHI 2005 Workshop on Awareness Systems, Portland, OR, US, ACM Press, New York (2005)

[3] Riche, Y., Mackay, W.: Peercare: Challenging the monitoring approach. In: B-HCI WShop on Designing Technology for the Older Population, Edinburg, UK (2005)

[4] Bly, S., Harrison, S., Irwin, S.: Mediaspaces: Bringing people together in a video, audio and computing environment. Comm. of the ACM 36(1), 28-47 (1993)

[5] Hutchinson, H., Mackay, W., Westerlund, B., Bederson, B.B., Druin, A., Plaisant, C., Beaudouin-Lafon, M., Conversy, S., Evans, H., Hansen, H., Roussel, N., Eiderback, B.: Technology probes: inspiring design for and with families. In: ACM SIGCHI 2003 Conf., Ft. Lauderdale, USA, pp. 17-24. ACM Press, New York (2003) 